\title{
Orquídeas del río Gaira, Sierra Nevada de Santa Marta, Colombia
}

\author{
Diego Yepes-Rapelo, Eduino Carbonó-Delahoz*, Michelle Pinto-Méndez \\ Herbario UTMC, Universidad del Magdalena, Santa Marta, Colombia
}

\begin{abstract}
Resumen
Como resultado de un estudio de las Orchidaceae en la cuenca del río Gaira en la Sierra Nevada de Santa Marta, este ensayo registra 115 especies distribuidas en 64 géneros, 19 subtribus y nueve tribus. La cantidad de especies supera el total de registros conocidos hasta ahora para la Sierra, a pesar de provenir de un área equivalente al $1 \%$ de su territorio. Epidendroideae, con 104 especies y 55 géneros, fue la subfamilia con mayor riqueza, en tanto que Pleurothallidineae se destacó como la más rica de las subtribus (22 especies y 11 géneros). De las especies presentes, 33,9 \% se distribuyó en siete géneros, siendo Epidendrum el más rico (13 especies), mientras que 64,04 \% de los géneros se registró como uniespecífico. Las especies epifíticas correspondieron a 77,39 \% en la región estudiada, $21,73 \%$ de las cuales era terrestre. Oncidium nevadense fue el único endemismo de la familia recolectado en la zona de estudio, que al igual que O. gloriosum y O. naevium se consideran especies amenazadas en el país.
\end{abstract}

Palabras clave: diversidad, epífitas, taxonomía, Orchidaceae.

\section{Orchids of the Gaira river, Sierra Nevada de Santa Marta, Colombia}

\begin{abstract}
As a result of a study of the Orchidaceae in the Gaira River basin, in the Sierra Nevada de Santa Marta, Colombia, this essay reports 115 species distributed in 64 genera, 19 subtribes and nine tribes. The number of species exceeds all known records so far for the Sierra, although they were collected in $1 \%$ of its area. Epidendroideae, with 104 species and 55 genera, was the richest subfamily; Pleurothallidinae stood out as the subtribe encompassing the largest number of species (22 species and 11 genera). Of the species present, 33.9\% were distributed in seven genera, Epidendrum being the richest with 13 , while $64.04 \%$ of the genera were monospecific. Epiphytic species equaled $77.39 \%$ in the territory and $21.73 \%$ of them were terrestrial. Oncidium nevadense was the only endemic species collected in the study area, and together with O. gloriosum and O. naevium they are considered endangered species in the country.
\end{abstract}

Key words: Diversity, epiphytes, taxonomy, Orchidaceae.

\section{Introducción}

Orchidaceae es una de las familias más ricas entre las plantas con flores, quizá la segunda después de las Asteraceae (Chase, et al., 2015), pues diferentes autores estiman que la integran entre 19.500 y 27.801 (Dressler, 2005, WCSP, 2011, Stevens, 2015, Royal Botanic Gardens, Kew, Missouri Botanical Garden - The Plant List, 2015). Varias hipótesis se han explorado en relación con esta exuberante especiación: la tendencia del grupo a presentar especializaciones morfológicas y ecológicas, la proliferación de linajes epifíticos, la fascinante variedad de estrategias de polinización, la obligada interacción con micorrizas, y los eventos de deriva genética combinados con episodios de selección (Dodson, 2003, Gravendeel, et al., 2004, Cozzolino \& Widmer, 2005, Tremblay, et al., 2005, Otero \& Flanagan, 2006, Ackerman, et al., 2007, Phillips, et al., 2012, McCormick \& Jacquemyn, 2013).
A medida que se avanza en los estudios y en la refinación de la información, los registros del tamaño de la familia Orchidaceae para Colombia han variado de 3.200 a 3.848 especies distribuidas entre 220 y 438 géneros (Ortíz, 1995, The Royal Botanical Garden, Kew - WCSP, 2011, Giraldo \& Betancur, 2011, Ordóñez \& MontesPulido, 2013, Ortíz \& Uribe, 2015, Bernal, et al., 2015). Cifras recientes señalan la existencia de 4.270 especies agrupadas en 274 géneros en el territorio colombiano (MADS \& UNAL, 2015), pero hay estimaciones creíbles de cantidades superiores, que se podrán confirmar solo cuando se complete un listado de la orquideoflora del país (Martínez \& Ortíz, 2010).

\footnotetext{
*Correspondencia:

Eduino Carbonó-Delahoz, eduinoc@yahoo.com

Recibido: 10 de junio de 2015

Aceptado: 21 de octubre de 2015
} 
La diversidad de orquídeas registrada para la Sierra Nevada de Santa Marta es baja cuando se compara con otras zonas de Colombia. Las estimaciones van de 72 especies, distribuidas en 38 géneros (Rangel \& Garzón, 1995), a 113 especies en 59 géneros (Bernal, et al., 2015). Aunque este macizo montañoso ha sido bastante visitado y explorado por naturalistas y botánicos desde inicios del siglo XIX (Carbonó \& Lozano-Contreras, 1997), en algunas áreas ha habido poco trabajo de recolección y su flora no se ha estudiado sistemáticamente; aun así, es frecuente la aparición de publicaciones de nuevas especies y registros a partir de muestras procedentes de esta región (Dalström, 2012, Alvear \& Almeda, 2014, Alvear, et al., 2015). La necesidad de conocer la magnitud y la diversidad de la orquideoflora presente en la cuenca hidrográfica del río Gaira en la Sierra Nevada de Santa Marta, motivó esta investigación, con cuya ejecución se pretendía obtener información de campo que permitiera elaborar un listado actualizado en nomenclatura y conceptos taxonómicos de las especies existentes como contribución al conocimiento de la composición florística local y del país.

\section{Materiales y métodos}

\section{Área de estudio}

La cuenca hidrográfica del río Gaira ocupa 104,6 km² en la vertiente nor-occidental de la Sierra Nevada de Santa Marta, departamento del Magdalena, Colombia. Se localiza entre $11^{\circ} 52^{\prime} 06^{\prime \prime}$ y $11^{\circ} 10^{\prime} 08^{\prime \prime}$ latitud norte, y $74^{\circ} 46^{\prime} 22^{\prime \prime}$ y $74^{\circ} 11^{\prime} 07^{\prime}$ " longitud oeste (Figura 1). Limita en el norte con la cuenca del río Manzanares, en el sur con la del río Toribio, en el este con la del río Guachaca y en el oeste con el mar Caribe. El curso del río corre en dirección este-oeste y tiene una longitud de $32,53 \mathrm{~km}$ desde su nacimiento en la cuchilla de San Lorenzo, a $2.750 \mathrm{msnm}$, hasta su desembocadura en el mar, cerca del área urbana del corregimiento de Gaira.

Los factores que inciden en la aparición de los cambios temporales y espaciales del clima a lo largo de la cuenca son diversos; entre ellos se destacan la variación altitudinal y la orientación con respecto a la circulación de vientos (PérezPreciado, 1984). Los climas presentes van del cálido árido, cálido seco, cálido húmedo y templado muy húmedo al frío muy húmedo, y el clima cálido húmedo abarca la mayor extensión (Tamarís-Turizo \& López-Salgado, 2006). En general, el régimen de distribución de la precipitación presenta tendencia unimodal-biestacional, climas secos y moderadamente húmedos (Rangel-Ch. \& Carvajal-C., 2012); el período lluvioso se presenta de mayo a noviembre y el de menor pluviosidad entre diciembre y abril, y la mayor precipitación se concentra en octubre y noviembre con un promedio mensual variable según la altitud.

Los siguientes son los tipos principales de vegetación: en la región tropical hay ecosistemas de bosques espinosos secos de Platymiscium pinnatum y Gyrocarpus americanus y bosques ralos-cardonales de Mimosa arenosa y Stenocereus griseus (hasta los $400 \mathrm{msnm}$ ), y ecosistemas de bosques dominados por Zygia longifolia y Virola sebifera (hasta los $1.100 \mathrm{msnm}$ ). En la región subandina (hasta los 2.500 msnm), los bosques están dominados por Cavendishia callista y Tovomita weddeliana, y en la zona alta (2.700 $\mathrm{msnm}$ ), por Clusia multiflora y Weinmannia pinnata (Rangel-Ch., 2012). Las zonas media y alta de la cuenca también se caracterizan por soportar selvas nubladas en donde es notorio un alto epifitismo (Cleef, et al., 1984),

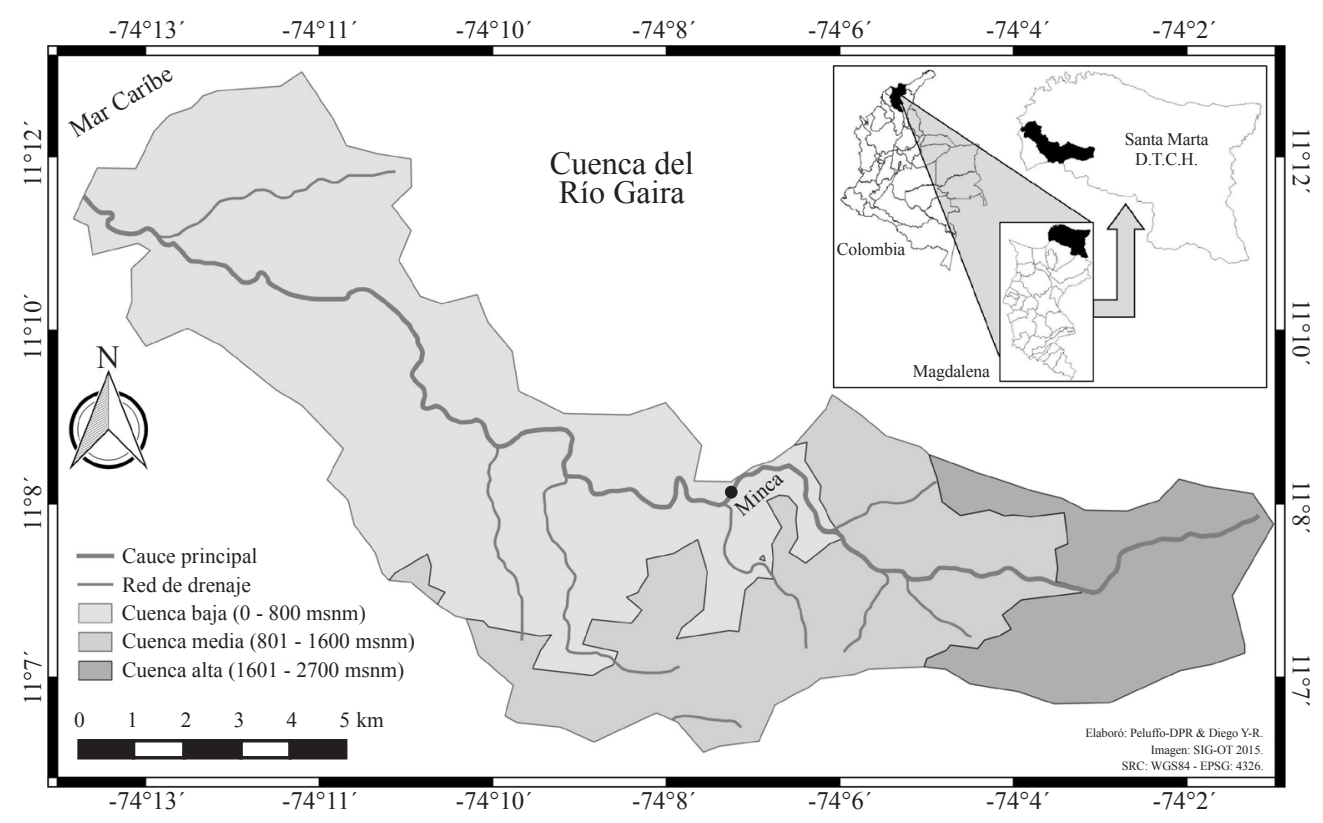

Figura 1. Localización de la cuenca del río Gaira, Sierra Nevada de Santa Marta, Colombia 
así como un número relativamente grande de especies de plantas de distribución restringida (Carbonó \& LozanoContreras, 1997, Alvear, et al., 2015).

\section{Muestreos y trabajo taxonómico}

Durante 2010 se hicieron recolecciones a lo largo de la cuenca del río Gaira. Algunas zonas se visitaron en distintas épocas para obtener la mayor cantidad de muestras en estado reproductivo. Para efectos del registro de la distribución altitudinal de las especies, la cuenca se subdividió en tres zonas: baja, con un rango altitudinal entre los 0 y los 800 msnm; media, entre los 800 y los 1.600 , y alta, entre los 1.600 y los 2.700 . Las muestras obtenidas se prensaron y procesaron de acuerdo con los estándares establecidos para preparar exicados, los cuales se depositaron en la colección del herbario UTMC.

La determinación se hizo mediante la consulta de información especializada y la revisión de las colecciones del Herbario Nacional Colombiano - COL, el de la Universidad Javeriana - HPUJ, el de la Universidad de los Andes ANDES y el de la Universidad del Magdalena - UTMC. Se contó con la asesoría de especialistas de las colecciones visitadas y la colaboración de orquidólogos del Botanischer Garten München-Nymphenburg, del Orchid Herbarium de Oakes Ames, de la Universidad de Harvard y de la Universidad del Valle.

El tratamiento taxonómico siguió, en general, la clasificación propuesta por Chase, et al. (2015) y en la subtribu Maxillariinae se conservaron los géneros propuestos por Blanco, et al. (2007). Para la circunscripción de taxones específicos se asumió el concepto morfológico de especie y para la identificación se siguieron discontinuidades en los caracteres, principalmente florales, como la posición del labelo y su relación con la columna, y la presencia, la forma y la cantidad de estructuras anexas (crestas o callos), así como los atributos vegetativos, de hábitat (terrestre o epífitas) y de distribución.

\section{Resultados}

En la cuenca del río Gaira se registraron 115 especies distribuidas en 64 géneros, 19 subtribus y nueve tribus de Orchidaceae. Las especies pertenecían a tres de las cinco subfamilias en que se considera subdividida la familia (Anexo, http://www.raccefyn.co/index.php/raccefyn/article/ downloadSuppFile/244/1182). El número de géneros correspondió a $23,25 \%$ del total registrado para Colombia, y el de las especies, a 2,69\%.

Las tribus representadas fueron las siguientes: Cymbidieae (siete subtribus, 29 géneros y 46 especies), Epidendreae (cinco subtribus, 21 géneros y 50 especies), Cranichideae (tres subtribus, seis géneros y siete especies), Sobralieae (dos géneros y cuatro especies), Vandeae (dos subtribus, dos géneros y tres especies), Orchideae (una subtribu, un género y dos especies), Malaxideae (una subtribu, un género y una especie), Vanilleae (un género y una especie) y Pogonideae (un género y una especie).

Las subtribus con mayor riqueza fueron Pleurothallidinae (11 géneros y 22 especies), Onciidinae (11 géneros y 20 especies) y Laellinae (siete géneros y 24 especies).

Los géneros más ricos fueron Epidendrum, con 13 especies, Pleurothallis, con seis, Stelis, con cinco, Oncidium, Prosthechea y Trichocentrum, con cuatro cada uno y Scaphyglottis, con tres.

De las especies encontradas en la cuenca del río Gaira, $77,39 \%$ eran epífitas, $21,73 \%$, terrestres y una $(0,86 \%)$, semiescandente. En la zona baja (0-800 msnm) se encontraron 30 especies, distribuidas en 26 géneros: cuatro terrestres y 25 epífitas y una semiescandente. Las subtribus más diversas en este rango altitudinal fueron Onciidinae y Laeliinae, con cinco géneros y siete especies cada una.

En la zona media (800-1.600 msnm) se recolectaron 37 especies de 27 géneros: cinco terrestres y 32 epífitas. Las subtribus de mayor riqueza fueron Laellinae (cuatro géneros y 10 especies) y Maxillarinae (cuatro géneros y cinco especies).

En la zona alta (1.600-2.700 msnm) se encontraron 48 especies distribuidas en 25 géneros: 15 terrestres y 33 epífitas. Las subtribus con más riqueza fueron Pleurothallidinae (nueve géneros y 17 especies), Onciidinae (tres géneros y seis especies) y Laellinae (dos géneros y seis especies).

Seis especímenes de los géneros Acianthera, Cyrtopodium, Dichaea y Epidendrum, no fueron identificados con certeza a nivel específico por no contar con evidencia suficiente para asignarles nombres.

Tres de las cuatro especies del género Oncidium que crecen en el río Gaira están catalogadas como especies bajo amenaza: Oncidium gloriosum, $O$. naevium y $O$. nevadense; la última de las tres es la única especie de Orchidaceae recolectada en este trabajo que se considera endémica para la Sierra Nevada de Santa Marta (Calderón-Sáenz, 2007) (Figura 2).

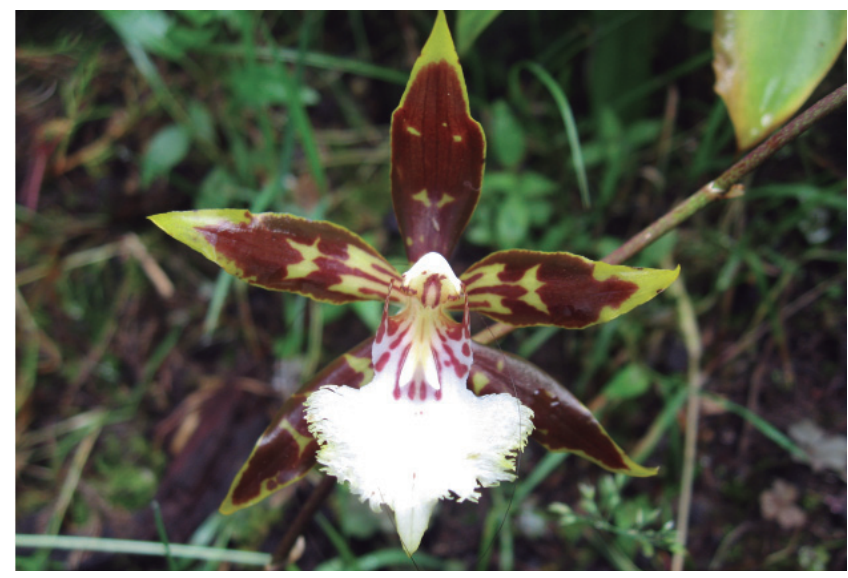

Figura 2. Flor de Oncidium nevadense 


\section{Discusión}

Las cifras de especies y géneros de Orchidaceae registradas en este trabajo superaron las estimadas y mencionadas para la Sierra Nevada de Santa Marta (Rangel-Ch. \& Garzón, 1995, Rivera Díaz \& Rangel-Ch., 2012, MADS \& UNAL, 2015); estos resultados, obtenidos en una porción del macizo montañoso equivalente a $1 \%$ de su extensión y en una altitud inferior a $3.000 \mathrm{~m}$, permiten conjeturar que la riqueza orquideológica registrada para la Sierra debe aumentar a medida que se amplíe la cobertura de la exploración en extensión y rango altitudinal, se intensifique el trabajo de recolección en zonas con escaso muestreo y se profundice el estudio del grupo en la región.

De las tres subfamilias presentes en el área de estudio, Epidendroideae tuvo casi la totalidad de la representación con 55 géneros $(85,93 \%)$ y 104 especies $(90,43 \%)$, lo que coincide con la riqueza del grupo en otras regiones tropicales. La subtribu Pleurothallidineae (EpidendroideaeEpidendreae) fue la más rica (11 géneros y 23 especies), característica que usualmente se presenta en el Neotrópico (Bogarín, et al., 2014).

Se registró un gran número de géneros uniespecíficos $(64,06 \%)$, condición que también se observó en el estudio de las orquídeas del valle geográfico del río Cauca (ReinaRodríguez, et al., 2010), y en los géneros de la familia en Colombia (MADS \& UNAL, 2015); sin embargo, es de destacar que Lepanthes y Masdevalia fueran uniespecíficos en la cuenca del Gaira cuando se cuentan entre los más ricos en el país (361 y 182 especies, respectivamente). Así mismo, siete de los géneros (Epidendrum, Pleurothallis, Oncidium, Prosthechea, Stelis, Trichocentrum y Scaphyglottis) acumularon 33,9 \% de las entidades específicas registradas, y el más rico, Epidendrum, correspondió a $11,3 \%$ de las especies, similar a lo observado para la familia en Colombia y en el Neotrópico.

Los resultados obtenidos indican afinidad con la flora orquideológica de Panamá. Si se exceptúan Cyrtochilum y Warreella, los demás géneros de Orchidaceae presentes en la cuenca del río Gaira se encuentran en ese país y 43 especies son compartidas (Bogarín, et al., 2014). En observaciones anteriores se había registrado una marcada relación de la flora del sudeste de Panamá con la de Colombia, particularmente con el departamento del Chocó (Bogarín, et al., 2013). Sin embargo, se puede reconocer una afinidad incluso mayor con la flora de orquídeas de Venezuela (Romero-González \& Carnevali FernándezConcha, 2000), pues 74 especies son compartidas, así como todos los géneros presentes en ese país. La cantidad de taxones comunes sería indicio de una relación fitogeográfica en la flora integrada por géneros de origen andino y mesoamericano. La semejanza observada, no obstante, puede atribuirse al rango altitudinal de este estudio (fajas de vegetación basal y subandina), en el cual no hay aislamientos marcados o interrupciones acentuadas entre las regiones del norte de Sudamérica comparadas, a diferencia de las franjas de vegetación de mayor altitud.

El 77,39 \% de las especies encontradas en la cuenca del río Gaira era epífita, lo que es frecuente en esta familia (Atwood, 1986); las observaciones efectuadas en otras regiones tropicales también señalan comportamientos parecidos al aquí registrado (Gravendeel, et al., 2004, Bogarín, et al., 2014). La evolución del epifitismo en las Orchidaceae se ha esgrimido como una de las fuentes de su diversidad, sin embargo, todavía no hay consenso sobre la explicación de las causas de la especiación en el grupo, por lo que se requieren renovados esfuerzos investigativos para su esclarecimiento (Tremblay, et al., 2005, Peakall, 2007, Philips, et al., 2012).

En las zonas media y alta de la cuenca del río Gaira se encontró la mayor riqueza de especies $(73,9 \%)$, lo que es explicable si se considera que la variación topográfica se ha asociado a la diversidad de orquídeas (Ackerman, et al., 2007; Ackerman, 2014). Esto se ajusta a los hallazgos de una mayor diversidad de la familia en elevaciones entre los 1.500 y los $2.800 \mathrm{~m}$ (Bogarín, et al., 2014), y coincide con lo observado para epífitos vasculares en Colombia, cuyo pico de riqueza se da entre los 1.700 y los $2.600 \mathrm{~m}$ (Krömer, et al., 2005), faja altitudinal donde hay heterogeneidad de hábitats que se convierten en espacios propicios, especialmente en los ambientes de las selvas montanas nubladas.

En la zona baja de la cuenca, hacia la desembocadura del río, la cobertura vegetal tiene rasgos marcadamente xerófilos, atribuibles a la escasez de precipitaciones y a la acción de vientos secantes casi todo el año; sin embargo, allí se registró la presencia de Encyclia cordigera, Caularthron bilamellatum, Dimerandra emarginata, Cohniella cebolleta, entre otras, a pesar de que se podía prever la ausencia de miembros de la familia en esas condiciones de baja humedad ambiental. Las especies destacadas se distribuyen a lo largo del corredor árido costero de la llamada zona seca del Caribe (CarbonóDelahoz, et al., 2013), y también están presentes en la costa de Venezuela (Leopardi, et al., 2009), lo que corrobora la capacidad de adaptación de las especies de Orchidaceae a entornos cálidos y secos (Ackerman, et al., 2007).

En desarrollo de este trabajo solo se recolectó una Orchidaceae endémica en la cuenca del Gaira (Oncidium nevadense), pero la contribución crítica de otros investigadores permitió observar la fotografía del tipo de Crossoglosa santamartana Ormerod (depositado en el herbario NY), y determinar la existencia de otro endemismo (Ormerod, 2013), que no se encontró durante la exploración de campo. A pesar de esta adición, el registro de especies exclusivas resultó bajo frente al número total de endemismos de orquídeas registrado para la Sierra Nevada de Santa Marta, en donde se han documentado 32 (MADS \& UNAL, 2015). Otras dos especies, no 
endémicas, Prescottia stachyodes (Sw.) Lind. y Dichaea dammeriana Kraenzlin, también se determinaron mediante la observación de fotografías de los tipos de la colección de H. H. Smith. Para la primera se tuvo acceso a la imagen del tipo de Prescottia smithii Schltr. Y a la referencia de Ayers \& Boufford (1988), y para la segunda, a la fotografía de un ejemplar rotulado como isosintipo, con lo que serían dos las adiciones a la lista general.

En general, las orquídeas tienen un valor ornamental que las hace apetecibles para coleccionistas y comerciantes, y las ha convertido en un grupo de plantas amenazadas (CalderónSáenz, 2007). Aunque la destrucción de hábitats debida a la intervención de los bosques constituye la amenaza principal, en la zona de estudio se extraen varias especies para ofrecerlas en venta, de manera que se requieren actividades puntuales de sensibilización sobre la conservación tanto de las especies como de los ambientes en los que crecen para contrarrestar el riesgo de afectar la estabilidad de la población de las orquídeas en la región.

Los estudios florísticos de las orquídeas en la Sierra requieren un mayor esfuerzo de recolección e investigación. Los resultados de la exploración de la cuenca del río Gaira indican que hay un conocimiento limitado de la flora de la región y que es necesario desplegar la capacidad requerida para entenderla a cabalidad. La recolección y el estudio en el ámbito local están plenamente justificados por la actualización del conocimiento sobre la distribución, la biología, la ecología, y la contracción y la expansión de poblaciones de la flora, que se logran con los hallazgos (Prather, et al., 2004). Algunas dificultades inherentes a las características del grupo exigen dedicación, especialmente en la preparación de los especímenes (Dressler, 2005), de manera que puedan ser de utilidad para los estudios de taxonomía de esta familia.

\section{Agradecimientos}

A la memoria del padre Pedro Ortíz Valdivieso, insigne investigador de las orquídeas de Colombia, de quien recibimos valiosos conocimientos y sabios consejos. Expresamos nuestro reconocimiento especial a Gustavo RomeroGonzález, Guillermo Reina-Rodríguez, Santiago Madriñán, Günter Gerlach y William Cetzal-Ix, por las valiosas recomendaciones e ingentes contribuciones científicas a este trabajo. Al Vicerrector de Investigaciones de la Universidad del Magdalena, José Henry Escobar, y a su equipo, por la siempre generosa y estimulante colaboración. A Héctor García-Quiñones, por el acompañamiento incondicional en todas las etapas del proyecto, y a los dos evaluadores anónimos cuyas correcciones, sugerencias e informaciones complementarias constituyeron aportes fundamentales para fortalecer el manuscrito.

\section{Conflicto de intereses}

Los autores declaran que no tienen conflicto de intereses.

\section{Bibliografía}

Ackerman, J. D., Trejo-Torres, J. C., Crespo-Chuy, Y. (2007). Orchids of the West Indies: Predictability of diversity and endemism. Journal of Biogeography. 34: 779-786.

Ackerman, J. D. (2014). Rapid transformation of orchid floras. Lankesteriana. 13 (3): 157-164.

Alvear, M. \& Almeda, F. (2014). Three new species of Monochaetum (Melastomataceae) from Colombia. Phytotaxa. 163 (1): 27-38

Alvear, M., Ocampo, G., Parra, C., Carbonó, E., Almeda, F. (2015). Melastomataceae of the Sierra Nevada de Santa Marta (Colombia): Floristic affinities and annotated catalogue. Phytotaxa. 195 (1): 1-30.

Atwood, J. T. (1986). The size of the Orchidaceae and the systematic distribution of epiphytic orchids. Selbyana. 9 (1): 171-186.

Ayers, T. J. \& Boufford, D. E. (1988). Index to the vascular plant types collected by H. H. Smith near Santa Marta, Colombia. Brittonia. 40 (4): 400-432.

Bernal, R., Gradstein, S. R., Celis, M. (editores). (2015). Catálogo de plantas y líquenes de Colombia. Instituto de Ciencias Naturales, Universidad Nacional de Colombia, Bogotá. Recuperado de: http://catalogoplantascolombia.unal.edu.co

Blanco, M. A., Carnevali, G., Whitten, W. M., Singer, R. B., Koehler, S., Williams, N. H., Ojeda, I., Neubig, K. M., Endara, L. (2007). Generic realignments in Maxillariinae (Orchidaceae). Lankesteriana. 7 (3): 515-537.

Bogarín, D., Pupulin, F., Arrocha, C., Warner, J. (2013). Orchids without borders: Studying the hotspot of Costa Rica and Panamá. Lankesteriana. 13 (1-2): 13-26.

Bogarín, D., Serracín, Z., Samudio, Z., Rincón, R., Pupulin, F. (2014). Un listado actualizado de las Orchidaceae de Panamá. Lankesteriana. 14 (3) - Supplement: 1-31.

Calderón-Sáenz, E. (editor). (2007). Libro Rojo de Plantas de Colombia. Volumen 6: Orquídeas, Primera Parte. Series Libros Rojos de Especies Amenazadas de Colombia. Bogotá, Colombia. Instituto Alexander Von HumboldtMinisterio de Ambiente, Vivienda y Desarrollo Territorial.

Carbonó, E. \& Lozano-Contreras, G. (1997). Endemismos y otras singularidades de la Sierra Nevada de Santa Marta, Colombia. Posibles causas de origen y necesidad de conservarlos. Rev. Acad. Colomb. Cienc. 21 (81): 409-419.

Carbonó-Delahoz, E., Barros-Barraza, A., Jiménez-Vergara, J. (2013). Cactaceae de Santa Marta, Magdalena, Colombia. Rev. Acad. Colomb. Cienc. 37 (143): 177-187.

Chase, M. W., Cameron, K. M., Freudenstein, J. V., Pridgeon, A. M., Salazar, G., van den Berg, C., Schuiteman, A. (2015). An updated classification of Orchidaceae. Botanical Journal of the Linnean Society. 177: 151-174.

Cleeef, A. M., Rangel Ch., O., van der Hammen, T., Jaramillo, R. (1984). La vegetación de las selvas del transecto Buritacá. En: Estudios de Sistemas Tropandinos 2. La 
Sierra Nevada de Santa Marta (Colombia) Transecto Buritacá-La Cumbre (van der Hammen \& Ruíz Editores). Berlin-Stuttgart: J. Cramer.

Cozzolino, S. \& Widmer, A. (2005). Orchid diversity: An evolutionary consequence of deception? Trends in Ecology and Evolution. 20 (9): 487-494.

Dalström, S. (2012). A new Cyrtochilum (Orchidaceae: Oncidiiinae) from Sierra Nevada de Santa Marta in Colombia. Lankesteriana. 12 (3): 143-145.

Dressler, R.L. (2005). How Many Orchid Species? Selbyana. 26: 155-158.

Dodson, C. H. (2003). Why are there so many orchid species? Lankesteriana. 7: 99-103.

Giraldo, G. \& Betancur, J. (2012). Guía de campo de las orquídeas de Santa María (Boyacá, Colombia). Serie de guía de campo del Instituto de Ciencias Naturales, Universidad Nacional de Colombia No. 9., 187 p.

Gravendeel, B., Smithson, A., Slik, F. J. W., Schuiteman, A. (2004). Epiphytism and pollinator specialization: Drivers for orchid diversity? Phil. Trans. R. Soc. Lond. B. 359: 1523-1535.

Kromër, T., M. Kessler, S. R. Grasdstein, A. Aceby (2005). Diversity patterns of vascular epiphytes along an elevation gradient in the Andes. Journal Biogeography. 32: 17991809.

Leopardi, C., Véliz, J., Cumana, L. J. (2009). Orquideoflórula preliminar de la península de Araya y áreas adyacentes, estado Sucre, Venezuela. Acta Botánica Venezuelica. 32 (1): $159-177$

Martínez, C. \& Ortiz, P. (2010). Orquídeas Especies de Colombia. Bogotá D. C.: Villegas Editores.

McCormick M. \& Jacquemyn, H. (2013). What constrains the distribution of orchid populations? New Phytologist. 202: $392-400$

MADS \& UNAL. (2015). Ministerio de Ambiente y Desarrollo Sostenible y Universidad Nacional de Colombia. Plan para el estudio y la conservación de las orquídeas en Colombia. Textos: Betancur, J., H. Sarmiento-L., L. Toro-González \& J. Valencia. Ministerio de Ambiente y Desarrollo Sostenible, Colombia; Universidad Nacional de Colombia, Bogotá D.C

Ormerod, P. (2013). Notes on Neotropical Liparoids (Orchidaceae: Malaxidae). Harvard Papers in Botany. 18: 39-49.

Ordóñez, J. \& Montez-Pulido, C. (2013). Orquideoflora de la reserva natural Quininí, Tibacuy, Cundinamarca, Colombia, y consideraciones para su bioprospección. Orquideología. 30 (1):18-33.

Ortíz, P. (1995). Orquídeas de Colombia. Santafé de Bogotá, Colombia. Corporación Capitalina de Orquideología.

Ortíz Valdivieso, P. \& Uribe Vélez, C. N. (2015). Orquídeas, tesoro de Colombia. Bogotá D.C.: Da Vinci Publicidad y Medios \& Cía. S en C.

Otero, J. T. \& Flanagan, N. S. (2006). Orchid diversity-beyond deception. Trends in Ecology and Evolution. 21 (2): 64-65.
Peakall, R. (2007). Speciation in the Orchidaceae: Confronting the challenges. Molecular Ecology. 16: 2834-2837.

Pérez Preciado, A. (1984). Aspectos climáticos de la Sierra Nevada de Santa Marta. En: Estudios de Sistemas Tropandinos 2. La Sierra Nevada de Santa Marta (Colombia) Transecto Buritacá-La Cumbre (van der Hammen \& Ruíz Editores). Berlin-Stuttgart, J. Cramer.

Phillips, R. D., Kingsley, K. W., Peakall, R. (2012). Low population genetic differentiation in the Orchidaceae: Implications for the diversification of the family. Molecular Ecology. 21: 5208-5220.

Prather, L. A., Álvarez-Fuentes, A., Mayfield, M. H., Ferguson C. J. (2004). Implications of the decline in plant collecting for systematic and floristic research. Systematic Botany. 29 (1): $216-220$.

Rangel-Ch., J. O. 2012. Ecosistemas del Caribe colombiano. En: Colombia Diversidad Biótica XII. La región Caribe de Colombia, Rangel-Ch. J. O. (editor). Universidad Nacional de Colombia-Instituto de Ciencias Naturales. p. 963-1009.

Rangel-Ch., J. O. \& Garzón, A. (1995). Sierra Nevada de Santa Marta (Colombia). En: J. O. Rangel-Ch. (editor), Colombia Diversidad Biótica. Vol. I. Instituto de Ciencias Naturales. p. $155-170$.

Rangel Ch. J. O. \& Carvajal-Cogollo, J. E. (2012). Clima de la región Caribe colombiana. En: Colombia Diversidad Biótica XII: la región Caribe de Colombia, Rangel, Ch., J. O. (editor). Bogotá: Instituto de Ciencias Naturales. p. 67-129.

Reina-Rodríguez, G. A., Ospina-Calderón, N. H., Castaño, A., Soriano, I., Otero, J. T. (2010). Catálogo de las orquídeas del valle geográfico del río Cauca y su piedemonte andino bajo, sur-occidente colombiano. Cespedesia. 32 (90-91): 7-22.

Rivera Díaz, O. \& Rangel-Ch., J. O. (2012). Diversidad de espermatofitos de la región Caribe colombiana. Colombia Diversidad Biótica XII: La región Caribe de Colombia, Bogotá: Instituto de Ciencias Naturales. p. 199-317.

Romero-González, G. A. \& Carnevali Fernández-Concha, G. (2000). Orchids of Venezuela: An Illustrated Field Guide. Second Edition. Armitano Editores. Caracas.

Stevens, P. F. (2015). Angiosperm Phylogeny Website. Version 13. Recuperado de: http://www.mobot.org/MOBOT/research/ APweb/. Último acceso el 20 de mayo de 2015.

Tamarís-Turizo, C. \& López-Salgado, H. (2006). Aproximación a la Zonificación Climática de la Cuenca del Río Gaira. Rev. Intropica. 3: 69-76.

Royal Botanic Gardens, Kew, Missouri Botanical Garden. The Plant List. (2015). Versión 1.1. Recuperado de: www. theplantlist.org. Último acceso el 27 de mayo de 2015.

Tremblay, R. L., Ackerman, J. D., Zimmerman, J. K., Calvo, R. N. (2005). Variation in sexual reproduction in orchids and its evolutionary consequences: A spasmodic journey to diversification. Biological Journal of the Linnean Society. 84: $1-54$

The Royal Botanical Garden, Kew. (2011). World Checklist of Selected Plant Families - WCSP. Recuperado de: http:// apps.kew.org/wcsp. Último acceso el 28 de mayo de 2015. 